\title{
Luvas cirúrgicas em procedimentos ortopédicos de trauma: Quantas perdem a integridade?*
}

\section{Surgical Gloves in Orthopedic Trauma Procedures: How Many Lose Their Integrity?}

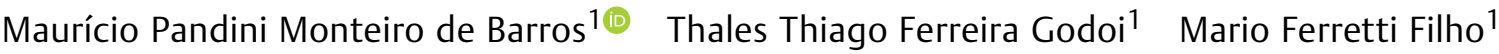 \\ Helio Jorge Alchavian Fernandes ${ }^{1}$ Fernando Baldy dos Reis ${ }^{1}$

\footnotetext{
${ }^{1}$ Departamento de Ortopedia e Traumatologia, Escola Paulista de Medicina, Universidade Federal de São Paulo (Unifesp), São Paulo, SP, Brasil
} \\ Endereço para correspondência Maurício Pandini Monteiro de \\ Barros, Rua: Correia de Lemos, 571, Apto. 142, CEP 04140-000, \\ Chácara Inglesa, São Paulo, SP, Brasil \\ (e-mail: mpmbarros@gmail.com).
}

Rev Bras Ortop 2021;56(3):379-383

\section{Resumo \\ Palavras-chave \\ - cirurgiões ortopédicos \\ - luvas cirúrgicas \\ - proteção \\ - traumatologia \\ Introdução Cirurgias ortopédicas apresentam a possibilidade de perfuração das luvas, que pode chegar a $56,8 \%$, relacionada principalmente à manipulação de instrumentos cortantes. O tratamento de fraturas e cirurgias de trauma apresenta risco adicional pelo contato com espiculas ósseas. \\ Objetivo Análise da prevalência de perda de integridade das luvas cirúrgicas em procedimentos ortopédicos de trauma, principalmente fraturas, avaliando a exposição do cirurgião e o contato com secreções provenientes do paciente. \\ Métodos Inspeção macroscópica das luvas de dois cirurgiões especializados em trauma, durante um período de 4 meses. Ambos usaram duas luvas para todos os procedimentos e, ao término da cirurgia, analisaram a presença ou ausência de manchas de sangue nas luvas internas e/ou nos dedos. Os procedimentos foram categorizados quanto ao tempo e tipo de cirurgia. A intercorrência investigada foi a perfuração de uma ou duas luvas; se a perfuração foi percebida imediatamente ou apenas ao final da cirurgia, e qual o local e o motivo do rasgo, se identificado. \\ Resultados Foram incluídas 210 cirurgias, das quais 87 apresentaram perfurações, sendo 17 casos em ambas as luvas e 70 apenas na luva externa. Um total de $27,5 \%$ dos danos foram descobertos apenas no final da cirurgia; os rasgos se concentraram no indicador esquerdo em $62,5 \%$ dos casos. Por último, houve uma relação mais significativa com cirurgias de foco aberto e com duração superior a 60 minutos. \\ Conclusão $\mathrm{O}$ nosso resultado sugere que em cirurgias prolongadas e com foco aberto, é necessário maior cuidado e inspeção à procura de danos nas luvas.}

Trabalho desenvolvido no Departamento de Ortopedia e Traumatologia, Escola Paulista de Medicina, Universidade Federal de São Paulo (Unifesp), São Paulo, SP, Brasil. recebido

22 de Junho de 2020

aceito

16 de Setembro de 2020
DOI https://doi.org/

10.1055/s-0040-1722591. ISSN 0102-3616. (c) 2020. Sociedade Brasileira de Ortopedia e Traumatologia. All rights reserved.

This is an open access article published by Thieme under the terms of the Creative Commons Attribution-NonDerivative-NonCommercial-License, permitting copying and reproduction so long as the original work is given appropriate credit. Contents may not be used for commercial purposes, or adapted, remixed, transformed or built upon. (https://creativecommons.org/ licenses/by-nc-nd/4.0/)

Thieme Revinter Publicações Ltda., Rua do Matoso 170, Rio de Janeiro, RJ, CEP 20270-135, Brazil 


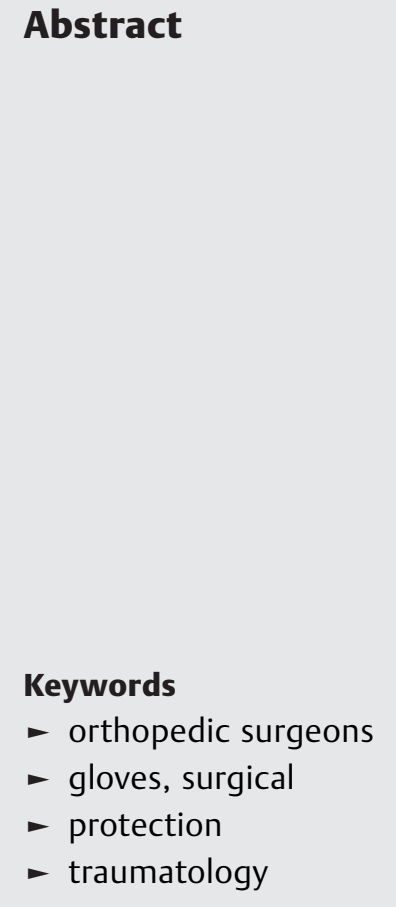

Introduction The possibility of perforation of gloves during orthopedic surgeries can reach $56.8 \%$, and it mainly related to the manipulation of blunt instruments. Surgeries for the treatment of fractures and trauma present additional risk due to contact with bone spires.

Objective Analysis of the prevalence of loss of integrity of surgical gloves in orthopedic trauma procedures, especially fractures, and evaluation of the surgeon's exposure and contact with secretions from the patient.

Methods Macroscopic inspection of the gloves of two surgeons specialized in trauma, over a period of 4 months. Both used two gloves for all procedures and, at the end of the surgery, analyzed the presence or absence of blood stains on the internal gloves and/or fingers. The procedures were categorized according to the time and type of surgery. The intercurrence investigated was the perforation of one or two gloves; if the tear was perceived immediately or only at the end of the surgery, and the location of and reason for the tear, if identified.

Results A total of 210 surgeries were included, 87 of which presented perforations, with 17 cases occurring in both gloves and 70 only in the outer glove. Finally, there was a more significant relationship with open focus surgeries and duration $>60$ minutes. Conclusion Our results suggest that greater care and inspection of gloves to look for damage are needed in prolonged surgeries with an open focus.

\section{Introdução}

Cirurgias ortopédicas apresentam maior possibilidade de perfurações nas luvas, devido à manipulação de instrumentais cortantes e afiados (brocas, parafusos, fios metálicos, entre outros), ${ }^{1}$ além de espículas ósseas que podem ferir a mão do cirurgião e oferecer um risco de contaminação.

Alguns estudos avaliaram a hipótese de aumento do risco de infecção nos procedimentos em que a luva do cirurgião perde a integridade, porém os resultados indicaram ausência de correlação. ${ }^{1-4}$

Perfurações das luvas ocorrem em $3,5^{5}$ a $56,8 \%{ }^{3}$ das cirurgias; em até $89 \%$ destas, o cirurgião pode não estar ciente da contaminação. ${ }^{6}$ Além disso, as perfurações se concentram no indicador e no polegar da mão não dominante. ${ }^{1}$

Utilizar duas luvas diminui em $87 \%$ a chance de contaminação do cirurgião em casos de rasgos; e, quando ocorre perfuração na mão do cirurgião por agulha sólida, há uma retenção de até $95 \%$ do sangue nas luvas, minimizando uma possível transmissão de doenças. ${ }^{1} \mathrm{O}$ par adicional de luvas diminui a possibilidade de perfuração da luva interna, reduzindo em até 13 vezes o risco de contaminação. ${ }^{7}$

Não há ainda estudos na literatura que avaliaram os rasgos das luvas somente em procedimentos de traumatologia ortopédica e correção de fraturas. 0 nosso objetivo é avaliar a exposição do paciente e do cirurgião, determinando a prevalência de perfurações de luvas neste tipo de cirurgia.

\section{Materiais e métodos}

Dois cirurgiões ortopédicos com especialização em trauma ortopédico, M. B. e T. G., com 4 e 5 anos de formação, respectivamente, ambos destros, inspecionaram as luvas durante e após as cirurgias, em um período de 4 meses, de $1^{\circ}$ de julho de 2019 a 30 de outubro de 2019, nos hospitais em que fazem rotina cirúrgica (Hospital Santa Cecília, Hospital Sancta Maggiore Mooca, Hospital Salvalus e Hospital Geral de Carapicuíba).

Os critérios de inclusão foram cirurgias de trauma ortopédico, em que um dos dois cirurgiões envolvidos nesse trabalho participou como cirurgião principal; procedimentos para correção e fixação de fraturas; retirada de materiais de síntese (placas ou hastes); revisões, e pseudartroses.

Os critérios de exclusão foram cirurgias de partes moles (reparos tendíneos ou ligamentares), sem uso de implantes ortopédicos, ou retiradas de materiais de síntese percutâneos.

Ambos os cirurgiões fazem rotineiramente o uso de dois pares de luvas (luva interna e luva externa). Todas as luvas usadas nos serviços e hospitais frequentados pela nossa equipe são de látex de borracha natural, de marcas autorizadas pelo órgão regulador de qualidade para uso cirúrgico estéril.

Ao final da cirurgia (se não houve perfuração percebida ao longo do procedimento e já feita a troca), foi retirada a luva externa de cada mão separadamente e inspecionada visual e minuciosamente em busca de sinais de manchas de sangue na luva interna (-Figura 1). 0 mesmo procedimento foi repetido com a luva interna, em busca de contaminação na mão do cirurgião (-Figura 1).

As cirurgias foram divididas em duração maior ou menor que 60 minutos. Além disso, dividimos em três grupos quanto ao tipo de cirurgia: percutâneas (como fixação de rádio distal com fio de Kirchner), cirurgias de foco fechado (como hastes intramedulares de tíbia ou fêmur com redução indireta), e 


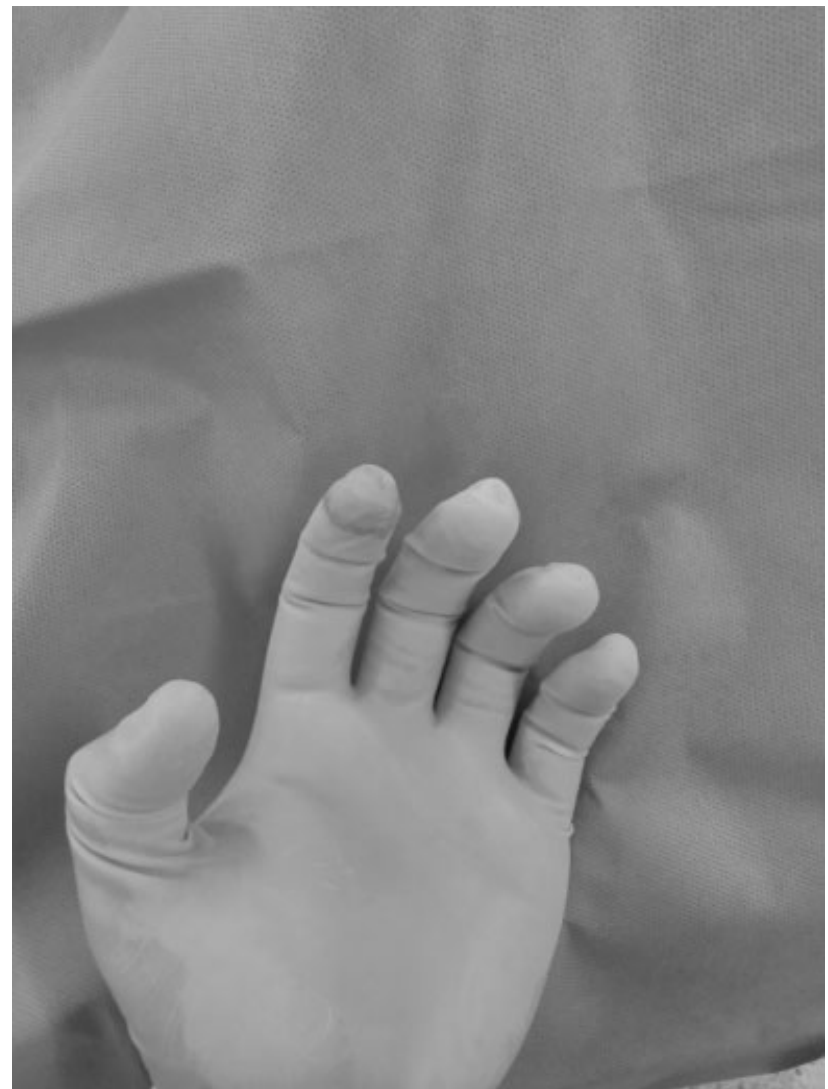

Fig. 1 luva interna mostrando perda da integridade do indicador esquerdo percebido ao final da cirurgia.

cirurgias de foco aberto (redução direta e manipulação dos fragmentos ósseos, como antebraço ou fraturas articulares)

Em relação à perda de integridade das luvas, separamos as luvas em dois grupos: quanto à perfuração e quanto aos detalhes; estes foram subdivididos da seguinte maneira:

Quanto à perfuração, os desfechos podem ser: Grupo A: "sem rasgos": não identificado perfuração durante o procedimento e, ao final, sem manchas nas luvas internas. Grupo B: "uma luva durante": perda da integridade apenas da luva externa notada durante o procedimento, porém sem danos à luva interna. Grupo C: "duas luvas durante": perda da integridade de ambas as luvas percebida durante o procedimento, com manchas na luva interna e mão do cirurgião. Grupo D: "uma luva final": apenas ao término da cirurgia foi notada mancha da luva interna, não sendo identificado o momento em que houve a perfuração. Por último o Grupo E: "duas luvas final": dano na luva interna e externa, com contaminação inclusive da mão do cirurgião, não notado durante o procedimento.

Quanto aos detalhes dividimos em: dedo e lado, registro quanto à região e lateralidade das perfurações; e Momento e modo, se percebido o momento e motivo do rasgo, como espículas ósseas, fios de Kirchner, durante manuseio do perfurador, ou ao posicionar afastadores do tipo Hohmann ou pinças de redução.

Ao final foi feito análise estatística das variáveis, usando teste qui-quadrado para comparação entre as diversas variáveis encontradas.
Tabela 1 Relação entre tempo de cirurgia e perfurações

\begin{tabular}{|l|l|l|l|}
\hline \multirow{2}{*}{} & \multicolumn{2}{|l|}{ Tempo cirúrgico } & total \\
\cline { 2 - 4 } & $<60$ minutos & $>\mathbf{6 0}$ minutos & \\
\hline Total & 116 & 94 & 210 \\
\hline $\begin{array}{l}\text { Luvas } \\
\text { danificadas }\end{array}$ & $24(20,6 \%)$ & $63(67,0 \%)$ & $87(41,4 \%)$ \\
\hline $\begin{array}{l}\text { Luvas } \\
\text { integras }\end{array}$ & $92(79,3 \%)$ & $31(32,9 \%)$ & $123(58,5 \%)$ \\
\hline
\end{tabular}

\section{Resultados}

Foram avaliados 210 procedimentos cirúrgicos envolvendo materiais de fixação interna, como fraturas, revisões, retiradas de material. Quanto ao tempo, 116 (55,2\%) duraram menos de 60 minutos, e 94 (44,7\%) duraram mais de 60 minutos. Quanto ao tipo de cirurgia, 20 cirurgias foram percutâneas, 60 de foco fechado e 130 de foco aberto. Em relação à perda de integridade, $41,4 \%$ dos procedimentos tiveram perfurações ou danos às luvas.

No que se refere ao tempo, os procedimentos com duração maior do que 60 minutos apresentaram maior índice de rasgos, contando $67 \%$. Já em cirurgias que duraram menos de 1 hora, houve $20,6 \%$ de perda da integridade $(p<0,001)$

\section{(-Tabela 1).}

Quanto ao tipo de cirurgia, os procedimentos de foco aberto se destacaram, com $49,2 \%$ deles apresentando perfurações. Por outro lado, 33,3\% das cirurgias de foco fechado e $15 \%$ das cirurgias percutâneas tiveram danificação de luvas $(p=0,005)$ (-Tabela 2).

Quanto aos desfechos, considerando as 87 cirurgias em que houve perfuração, a situação de perceber rasgo apenas na luva externa (grupo B) durante a cirurgia teve maior índice, contando com $24,2 \%$. Já os casos em que houve perda da integridade de duas luvas notada durante a cirurgia (grupo C) corresponderam a 5,7\%. Dentre os procedimentos em que a ocorrência de rasgo foi descoberta apenas ao final da cirurgia, $9 \%$ ocorreram apenas na luva externa (grupo D) e 2,3\% em ambas as luvas (grupo E) (-Tabela $\mathbf{3}$ ).

Em relação à percepção, quando houve rasgos, 63 casos foram identificados no momento ocorrido ou ainda durante a cirurgia. Por outro lado, 24 das perfurações foram notadas apenas ao final da cirurgia durante a inspeção. Em $80 \%$ dos casos em que houve rasgo, a luva interna se manteve intacta,

Tabela 2 Relação entre tipo de cirurgia e perfurações

\begin{tabular}{|l|l|l|l|l|}
\hline \multirow{2}{*}{} & \multicolumn{3}{|l|}{ Tipo de cirurgia } & total \\
\cline { 2 - 5 } & Percutânea & $\begin{array}{l}\text { Foco } \\
\text { fechado }\end{array}$ & $\begin{array}{l}\text { Foco } \\
\text { aberto }\end{array}$ & \\
\hline Total & 20 & 60 & 130 & 210 \\
\hline $\begin{array}{l}\text { Luvas } \\
\text { danificadas }\end{array}$ & $3(15 \%)$ & 20 & 64 & 87 \\
\hline $\begin{array}{l}\text { Luvas } \\
\text { integras }\end{array}$ & $17(85 \%)$ & $\begin{array}{l}40 \\
(66,6 \%)\end{array}$ & $\begin{array}{l}66 \\
(59,7 \%)\end{array}$ & $\begin{array}{l}(58,5 \%) \\
(53), 3 \%)\end{array}$ \\
\hline
\end{tabular}


Tabela 3 Relação à percepção das perfurações

\begin{tabular}{|l|l|l|l|l|}
\hline \multicolumn{2}{|l|}{$\begin{array}{l}\text { Perfurações - percep- } \\
\text { ça }\end{array}$} & & & \\
\hline Grupo A & Grupo B & Grupo C & Grupo D & Grupo E \\
\hline $\begin{array}{l}123 \\
(58,5 \%)\end{array}$ & $51(24,2 \%)$ & $12(5,7 \%)$ & $19(9,0 \%)$ & $5(2,3 \%)$ \\
\hline Total & $87(41,4 \%)$ & & & \\
\hline
\end{tabular}

servindo como barreira para o contato direto entre paciente e cirurgião.

O local em que mais houve perfurações foi o indicador da mão não dominante (esquerda), com 62,5\% dos casos, seguido do indicador direito, com 19,2\%, e em terceiro, o polegar esquerdo, contando com 9,6\% (- Tabela 4).

Nos casos de perda de integridade em que o motivo foi identificado, o maior fator responsável foi espículas ósseas, com $45 \%$, seguido de perfurações com fios de Kirchner ou fios guia, com 22,5\%. Rasgos durante manuseio do perfurador foram o agente causador em $12,5 \%$, e o ato de posicionar um afastador do tipo Hohmann ou pinça de redução em $10 \%$ (-Tabela 5).

\section{Discussão}

Na literatura, encontramos análises diferentes quanto ao tema. Alguns autores, como Nicolai et al. ${ }^{8}$ e Chan et al., ${ }^{5}$ avaliaram as luvas da equipe cirúrgica, chegando em porcentagens de $14,6 \%$ e 3,5\%, respectivamente. Laine e Aarnio ${ }^{7}$ e Sanders et al. ${ }^{2}$ obtiveram números maiores analisando apenas as luvas dos cirurgiões, com $31,4 \%$ e $52 \%$ de perfurações, respectivamente. Já no nosso trabalho, encontramos uma prevalência de perfuração de $41,4 \%$.

O tempo cirúrgico é um fator claramente relacionado com a perda da integridade das luvas. Louis et al. ${ }^{9}$ indicaram que $90 \%$ das perfurações se concentram em procedimentos de mais de 2 horas de duração. Enz et al. ${ }^{10}$ também encontraram mais perfurações em revisões de artroplastia, que, portanto, tinham duração em média de 116 minutos. Laine e Aarnio ${ }^{7}$ indicaram a diferença de $3,6 \%$ de rasgos em cirurgias com menos de 1 hora para $14,6 \%$ naquelas de mais de 1 hora. Sanders et al. ${ }^{2}$ ainda afirmaram que na análise das luvas em procedimentos com mais de 3 horas, $100 \%$ apresentaram perfurações. Nosso artigo indicou uma diferença de $20,6 \%$ de rasgos em procedimentos mais curtos para $67 \%$ em cirurgias longas.

Chan et al., ${ }^{5}$ apesar de não incluir somente procedimento do trauma ortopédico, analisaram seus resultados separando também quanto ao tipo de cirurgia. Seu resultado apresentou maior índice de perfurações em procedimentos de fixação com hastes intramedulares, indicando 33\% de perfurações, seguido de $19 \%$ de rasgos em cirurgias com redução aberta. Divergindo desta informação, nosso trabalho em fixações internas com foco aberto apresentou 49,2\% de perfurações; já em procedimentos de foco fechado com hastes intramedulares, o resultado foi semelhante aos de Chan et al., ${ }^{5}$ com 33,3\%.

Loius et al. $^{9}$ e Mafulli et al. ${ }^{6}$ tiveram $80 \%$ e $89 \%$ das perfurações notadas apenas ao final da cirurgia, respectivamente; Laine e Aarnio ${ }^{7}$ indicaram $23 \%$ de percepção intraoperatória quando utilizando apenas uma luva e $36 \%$ com duas luvas. Nicolai et al. ${ }^{8}$ apresentaram percepção em $10,2 \%$ dos casos no grupo com luvas convencionais. Nosso artigo obteve um resultado inverso em relação a esses valores; obtivemos $72,4 \%$ de identificação intraoperatória das perfurações. Além disso, concluímos que o uso de dupla luva protegeu a mão do cirurgião em $80,4 \%$ dos procedimentos em que houve perda de integridade da luva; nesses casos, a luva interna permaneceu sem danos, assim como Tanner e Parkinson ${ }^{1}$ indicaram como fator protetor.

Quanto à localização das perfurações, Nicolai et al., ${ }^{8}$ Laine e Aarnio $^{7}$ indicaram ocorrência de $73,6 \%$ e $70 \%$ dos rasgos na mão não dominante; nossa análise encontrou um valor semelhante, com 76,6\%. Lee et al. ${ }^{11}$ encontraram uma distribuição de maior prevalência de furos no indicador não dominante, seguido do indicador dominante e o polegar não dominante, dados que estão de acordo com nossos resultados. Nosso artigo identificou $62,5 \%$ dos rasgos localizados no indicador da mão esquerda, $19,2 \%$ no indicador direito e $9,6 \%$ no polegar esquerdo.

Como limitação do presente trabalho, podemos mencionar que o método de detecção da perda de integridade difere daquele reconhecido e padronizado nos Estados Unidos e na Europa (The American Society for Testing and Materials e The European Standards Committee), que é o enchimento da luva

Tabela 4 Distribuição quanto ao local das perfurações

\begin{tabular}{|c|c|c|c|c|c|c|c|}
\hline \multicolumn{7}{|c|}{ Perfurações - local (dedo) } & \multirow[t]{2}{*}{ Total } \\
\hline $\begin{array}{l}\text { Indicador } \\
\text { esquerdo }\end{array}$ & $\begin{array}{l}\text { Indicador } \\
\text { direito }\end{array}$ & $\begin{array}{l}\text { Polegar } \\
\text { esquerdo }\end{array}$ & $\begin{array}{l}\text { Polegar } \\
\text { direito }\end{array}$ & $\begin{array}{l}\text { Anular } \\
\text { esquerdo }\end{array}$ & $\begin{array}{l}\text { Dedo médio } \\
\text { esquerdo }\end{array}$ & $\begin{array}{l}\text { Região } \\
\text { palmar }\end{array}$ & \\
\hline $65(62,5 \%)$ & $20(19,2 \%)$ & $10(9,6 \%)$ & $4(3,8 \%)$ & $\begin{array}{l}3 \\
(2,8 \%)\end{array}$ & $\begin{array}{l}1 \\
(0,9 \%)\end{array}$ & $1(0,9 \%)$ & 104 \\
\hline
\end{tabular}

Tabela 5 Motivo das perfurações

\begin{tabular}{|l|l|l|l|l|l|l|}
\hline Perfurações - motivo (quando) identificado & Total \\
\hline $\begin{array}{l}\text { Espículas } \\
\text { ósseas }\end{array}$ & $\begin{array}{l}\text { Fio de Kirchner/ } \\
\text { fio guia }\end{array}$ & Perfurador & $\begin{array}{l}\text { Posicionar Hohmann ou } \\
\text { pinça de redução }\end{array}$ & Sovela & $\begin{array}{l}\text { Palpar parafuso para } \\
\text { encaixar chave }\end{array}$ & \\
\hline $18(45 \%)$ & $9(22,5 \%)$ & $5(12,5 \%)$ & $4(10 \%)$ & $2(5 \%)$ & $2(5 \%)$ & 40 \\
\hline
\end{tabular}


com $1.000 \mathrm{ml}$ de água e a suspensão com um clamp pelo colarinho, permitindo que a água flua por possíveis perfurações. Outra opção encontrada na literatura é encher a luva com $500 \mathrm{ml}$ de água e espremê-la, avaliando o vazamento de água. ${ }^{12}$ Outra limitação foi o não detalhamento da população ou do tipo de cirurgia.

\section{Conclusão}

Nosso trabalho identificou $67 \%$ de perfurações em cirurgias mais prolongadas contra $20,6 \%$ em cirurgias de menos de uma hora. As cirurgias de redução aberta apresentaram perda da integridade em $49,2 \%$ dos casos, as de redução fechada em $33,3 \%$ dos casos, ao passo que as percutâneas apresentaram perda da integridade em apenas $15 \%$ dos casos. O dedo mais acometido foi o indicador da mão não dominante, responsável por $62,5 \%$ das perfurações. Além disso, em $72,4 \%$ das vezes, o rasgo foi percebido ao longo da cirurgia e a razão mais frequentemente observada foi o contato com espiculas ósseas.

\section{Conflito de interesses}

Os autores declaram não haver conflito de interesses.

\section{Referências}

1 Tanner J, Parkinson H. Double gloving to reduce surgical crossinfection. Cochrane Database Syst Rev 2006;2006(03):CD003087

2 Sanders R, Fortin P, Ross E, Helfet D. Outer gloves in orthopaedic procedures. Cloth compared with latex. J Bone Joint Surg Am 1990;72(06):914-917
3 Sebold EJ, Jordan LR. Intraoperative glove perforation. A comparative analysis. Clin Orthop Relat Res 1993;(297):242-244

4 Hayes G, Singh A, Gibson T, et al. Influence of orthopedic reinforced gloves versus double standard gloves on contamination events during small animal orthopedic surgery. Vet Surg 2017;46 (07):981-985

5 Chan KY, Singh VA, Oun BH, To BH. The rate of glove perforations in orthopaedic procedures: single versus double gloving. A prospective study. Med J Malaysia 2006;61(Suppl B):3-7

6 Maffulli N, Capasso G, Testa V. Glove perforation in elective orthopedic surgery. Acta Orthop Scand 1989;60(05):565-566

7 Laine T, Aarnio P. Glove perforation in orthopaedic and trauma surgery. A comparison between single, double indicator gloving and double gloving with two regular gloves. J Bone Joint Surg $\mathrm{Br}$ 2004;86(06):898-900

8 Nicolai P, Aldam $\mathrm{CH}$, Allen PW. Increased awareness of glove perforation in major joint replacement. A prospective, randomised study of Regent Biogel Reveal gloves. J Bone Joint Surg Br 1997; 79(03):371-373

9 Louis SS, Steinberg EL, Gruen OA, Bartlett CS, Helfet DL. Outer gloves in orthopaedic procedures: a polyester/stainless steel wire weave glove liner compared with latex. J Orthop Trauma 1998;12 (02):101-105

10 Enz A, Klinder A, Mittelmeier H, Kundt G, Mittelmeier W, Zaatreh S. Damages with High Consequences: Analysis of Perforations in Surgical Latex Operation Gloves from Orthopedic Surgeries. Eur J Microbiol Immunol (Bp) 2018;8(04):159-162

11 Lee SW, Cho MR, Lee HH, Choi WK, Lee JH. Perforation of Surgical Gloves during Lower Extremity Fracture Surgery and Hip Joint Replacement Surgery. Hip Pelvis 2015;27(01):17-22

12 Hester RA, Nelson CL, Harrison S. Control of contamination of the operative team in total joint arthroplasty. J Arthroplasty 1992;7 (03):267-269 